\title{
Coordination chemistry of graphite
}

\author{
M.E.Vol'pin and Yu.N.Novikov \\ Institute of Organo-element Compounds Academie of Sciences \\ of the USSR, Moscow, USSR
}

\begin{abstract}
The aromatic system of graphite is capable to form complexes with transition metals. The paper summarise the methods of preparation and catalytic properties of graphite compounds with iron, cobalt, nickel, molybdenum, palladium and other metals. The investigation by different physical methods including ExAFs proofs the $\pi$-complexes formation by the interaction of metal atoms with graphite. The comparison was carried out of theoretical calculations and experimental data.
\end{abstract}

The general trend of modern coordination chemistry is to spread its ideas and methods to the new fields - from solid state chemistry to the chemistry of living objects. Another feature of modern coordination chemistry is the growing attention to the polynuclear and eluster structures and to the structures with polyligands. Among the latter quasitwodimensial polyligands such as molybdenum and tungsten halcogenides, layered silicates and, especially, graphite are of significant importance.

Graphite and other carbon materials (pyrographite, pyrocarbon, amorphous carbon, carbon and graphite fibers and exfoliated graphite) are widely used in modern industry. The properties of such materials lie somewhere between those of metals, organic polymers and ceramies. We mean here such properties as strength, conductivity and density. But carbon materials have some properties which are superior compared to most of metals. These are thermostability high heat of evaporation and their ability to increase the strength with temperature.

The main structural units of the carbon materials are the graphitic domens included into the amorphous carbon phase. All the properties of different materials depend strongly on the size and mutual orientation of these domens and on the relative quantities of graphite and amorphous phases. One of the routes towards new carbon materials implies modification of the graphitic phase and first of all, their intercalation by inorganic species resulting in the formation of coordination compounds. Graphite intercalation compounds (GICs) are the promising type of coordination compounds.

It is well known that graphite fromework is built of carbon layers separated by $3.35 \AA$, which is approximately equal to the Van-der-Waals distance between carbon atoms. The in-plane C-C distance is $T .4 \uparrow \AA$. This is the same as $C-C$ bond length in aromatic hydrocarbons. Due to the weak interaction between carbon layers different substances can easy extend them and penetrate into the interlayer space thus forming the monomolecular layer. One can roughly classify GICs by the nature of the intercalant and Its interaction with the host matrix.

The intercalation of halogens, strong protic acids or aprotic acids, e.g. metal halogenides, leads to the formation of the acceptor type GICs. In this case some charge transfer occurs from carbon layers to the lower antibonding orbitals of the intercalated species. The carbon layers become positively charged. Such compounds are thus donor-acceptor complexes, the graphite being the electron donor. Significant p-type conductivity is observed in such GICs which in some cases (graphite-AsF for example) can achieve that of copper or even silver (ref.1). The interlayer interactions of the guest species result in very interesting magnetic properties of the acceptor-type GICs. For example, the first stage graphite $-\mathrm{FeCl}_{3}$ compound, in which the neighbouring 
intercalant layers are separated by one carbon layer, is antiferromagnetic as in the case of pure FeCl $1_{3}$. From the other hand, the higher stages GICs, where two salt layers are separated by 2 or more carbon layers, are ferromagnetic (refs 2-4). That means that one can synthesize different anisotropic magnetic materials by monitoring the layers stacking of one and the same guest substance.

The interaction of graphite with alkali and alkali-earth metals in vapour or liquid state leads to the formation of GICs in which metal atoms donate their electrons to the carbon layers. In this case the reverse donor-acceptor complexes are formed with negatively charged carbon layers and metalic cations between them. Such compounds exibit significant n-type (electron) conductivity. The in-plane conductivity in this GICs increases for several orders of magnitude and achieves that of aluminium, and (in the case of graphite fibers) even copper.

The graphite coordination compounds of the third type are the graphite complexes with the transition metals e.g. Cr, Mo, W, Mn, Fe, Co, Ni, Cu, Pd, Pt etc. Usually they are obtained by the reduction of metal chloride GICs with different electron donors: aromatic anion-radicals, sodium in liquid ammonia, metallic potassium, electron current etc. (refs 5-23). The compounds obtained are very stable against air, water and temperature.

What is the structure of transition metals GICs? This is a very intrigue question. By analogy with molecular $\pi$-complexes of aromatic, olefin and allyl type one could consider some $\pi$-interaction between metal atoms and carbon macromolecules due to the aromatic nature of carbon layers. Really, the evidence of such interaction in transition metal - graphite compounds have found in the MOssbauer and X-ray spectroscopy, diffraction and EXAFS studies of GICs.

For example, all the above methods prove unambiguously that the structure of GICs with VI group metals - Cr and Mo is of the sandwich type (refs 5, 9) like that of dibenzene chromium (Figure 1). The thickness of the field layers in GICs with $\mathrm{Cr}$ and Mo are $3.2 \AA$ and $3.75 \AA$, respectively, which is very close to the double metal-Iigand distance in aromatic complexes of these metals $3.22 \AA$ and $3.8 \AA$, respectively.

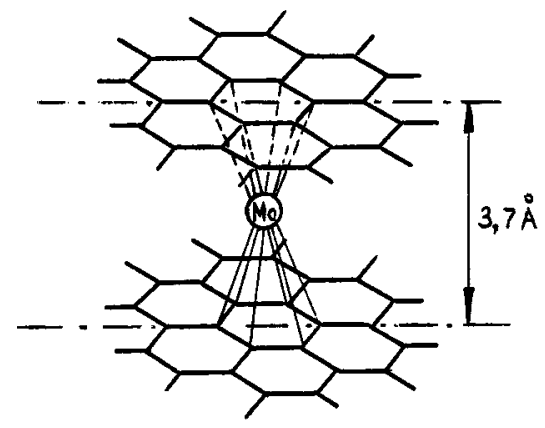

Fig. 1. Structural unit of Mo-GIC.

The GIC with Pd has a different structure. The filled layer thickness there is $4.3 \AA$ which is equal to the distance between benzene molecules in $\left(\mathrm{C}_{6} \mathrm{H}_{6}\right)_{2} \mathrm{Pd}_{2}\left(\mathrm{Al}_{2} \mathrm{Cl}_{8}\right)$. That means that carbon layers in $\mathrm{GIC}$ with $\mathrm{Pd}$ behave as both olefin and dien ligands, palladium atoms being in the oxidation state + 1. The latter conclusion is in an agreement with ESCA data (ref. 24).

The problem of the GIC with Fe, Co, NI and Mn structure is more complicated. The Mossbauer and X-ray spectroscoplc studies of GIC with Fe show that there are two types of iron in such compounds: atomic Fe in the interlayer spaces which (according to the Mossbauer spectra) form $\pi$-complexes with carbon layers and $\alpha-F e$ clusters which do not intercalate into graphite (refs 9,11, $15,25)$. The relation between these forms depends on the reduction conditions and in the favorable cases more than 908 of the iron is found in the form of

$\pi$-complexes. The Molssbauer and X-ray spectroscopy studies of GICs with Co and $\mathrm{Mn}$ also show that the metal atoms in these compounds form $\pi$-complexes with carbon layers (ref 26). Finaliy the structure of such GICs was determined by use of EXAFS method (ref.27) 

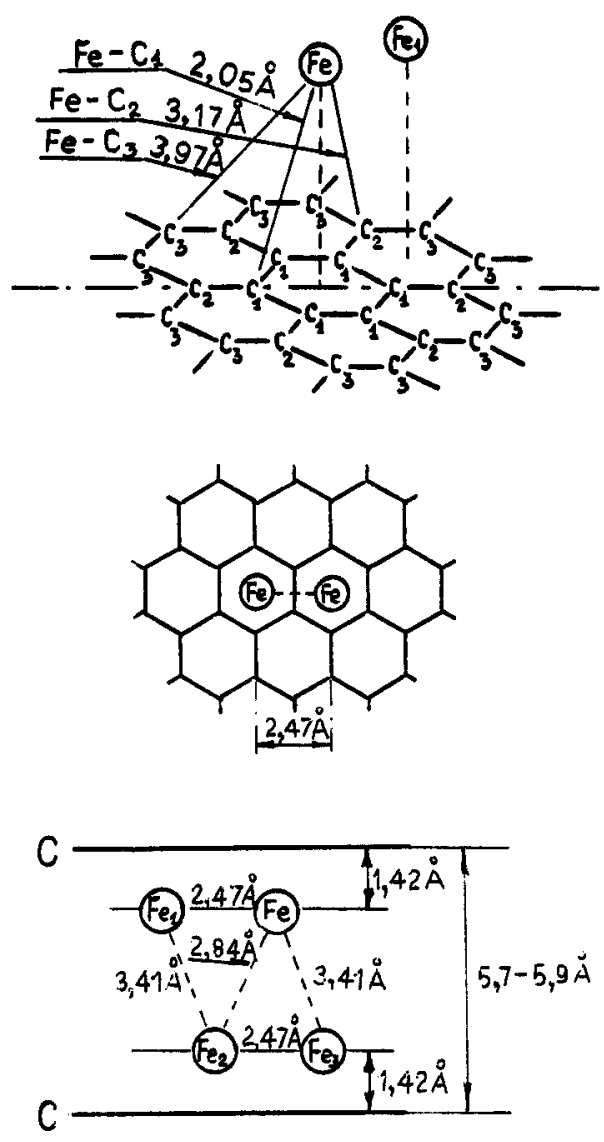

Fig.2. The structural unit of Fe-GIC based on EXFS's data. (a) orientation of the dimers $\mathrm{Fe}_{2}$ relative to the carbon plane (b) . Proection of the dimer $\mathrm{Fe}$ on the carbon plane (c). Orientation of $\mathrm{Fe}_{4}$ clusters in $\mathrm{Fe}-\mathrm{GIC}$.

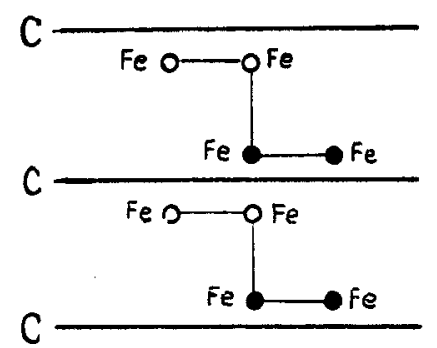

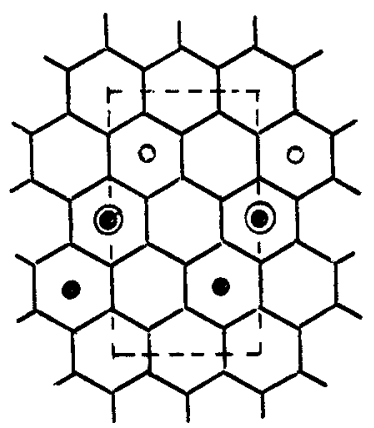

Fig.3. Structural model used in the Fe-GIC calculation (Ref.28). (a) Side proection. (b) Proection on the carbon plane.

It was found that in the GIC with Fe the iron atoms form bilayered structure within the interlayer space. The Fe atoms in each sublayer form dimers which are parallel to the carbon layers and are positioned at 1.42 A against the adjacent hexagon centers of the neighbouring carbon layers (Figure 2). The $\mathrm{Fe}-\mathrm{C}_{1}, \mathrm{Fe}-\mathrm{C}_{2}$ and $\mathrm{Fe}-\mathrm{C}_{3}$ distances are $7.00,3.20$ and $3.97 \mathrm{~A}$, respectively, the Fe-C distance being elose to that in ferrocene $(2.05 \AA)$. That means that the nature of the interaction between $\mathrm{Fe}$ and carbon layer and $\mathrm{Fe}$ and cyclopentadienyl ring could be similar. The separation of Fe atoms from the other carbon layer is significantly larger and hence there is no bonding between them.Besides Fe-C distance the EXAFS spectra provide information about Fe-Fe distance. One of the latter, namely $2.47 \AA$, can be assigned to the intradimer Fe-Fe distance and is also equal to the distance between the centers of the adjacent carbon hexagons (Figure $1 \mathrm{~b}$ ). Two other distances (2..82A and 3.4.1A) correspond to the $\mathrm{Fe}$ atoms in different dimers being at the neighbouring graphite layers. All these data result in the structutal model shown on the Figure 2. To conclude, Mossbauer, $X$-ray spectroscopy and EXAFS support the formation of $\pi$-complexes of transition metal atoms and carbon layers. So the next problem is the nature of the Fe-C bonding in such complexes.

The theoretical calculations of the electronic structure of Fe-GIC were made using two different approximations. In the first approximation one consider $\mathrm{Fe}_{2}$ dimer and naphtalene molecule, the latter taken instead of graphite layer. The Fe atoms are site above the centres of the six-membered rings parallel to the aromatic plane. The $C-C$ bonds and $C-C-C$ angles are taken as in graphite 
TABLE. The orbital populations of iron atoms

$\begin{array}{lll}\text { System Density of states } & \begin{array}{l}\text { Charge of } \\ \text { Fe atoms }\end{array}\end{array}$

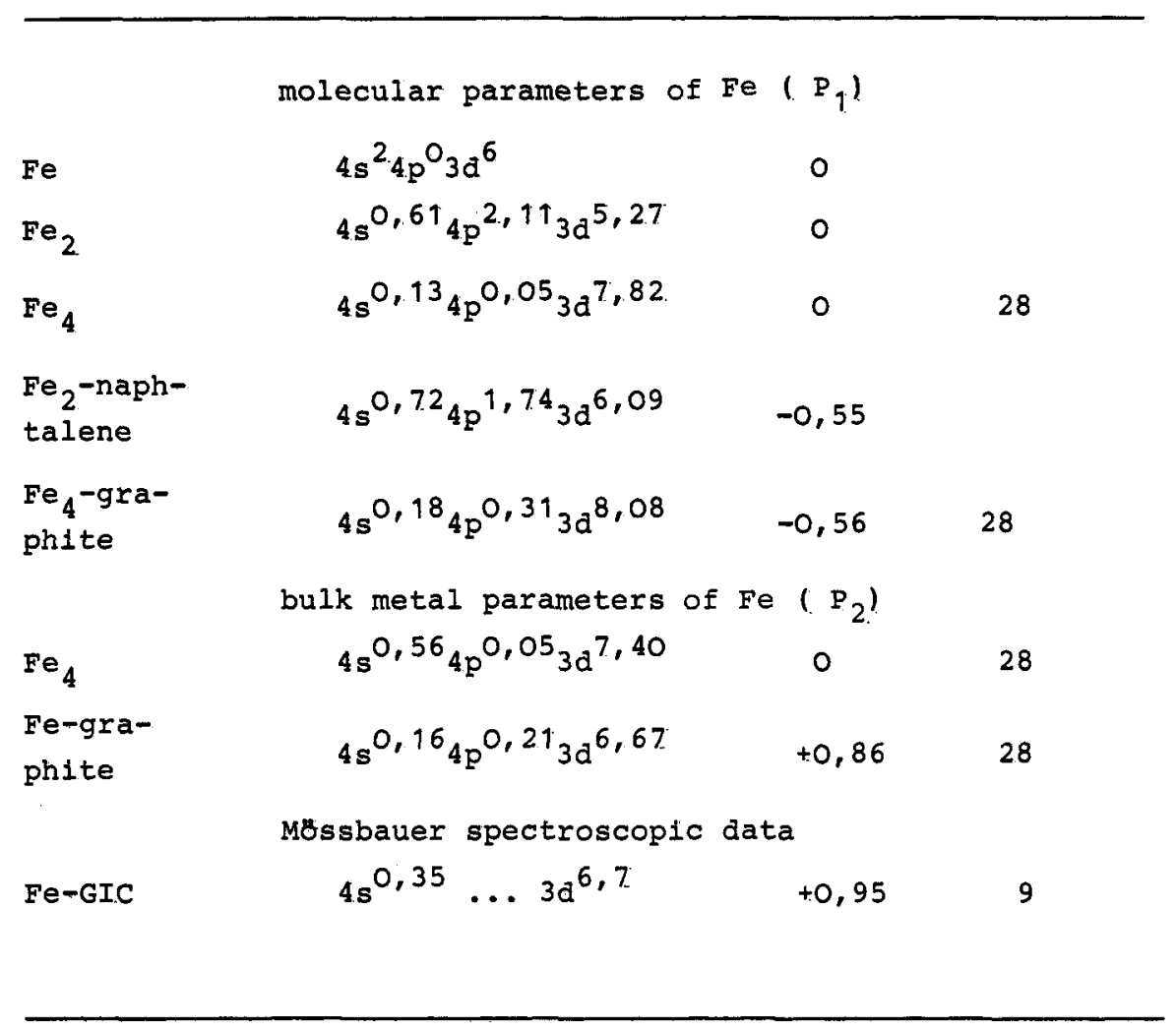

and Fe-Fe bond is assumed to be $2.46 \AA$ as in GIC with Fe. I.Stankevich and A.Chistyakov in our Institute made such calculations by use CNDO method for singlet, triplet and pentaplet Fe states. The variable parameter (R) was the distance between $F e$ atoms and naphthalene plane. The analysis of potential curves showed that the ground state of $F e$ is triplet and the equilibrium value of $R$ is $1.43 \AA$. According to EXAFS, this is very close to the same distance in GIC with Fe. So the dimers Fe, can form $\boldsymbol{\pi}$-complexes with naphthalene and probably with graphite layers. It is rather interesting that the formation of $\mathrm{Fe}_{2}$-naphthalene complex is accompanied by the large increase of 3d and decrease of $4 p$ electron density at the Fe site. The $4 \mathrm{~s}$ orbitals population remains practically the same (Table).

The alternative approximation deals with the three-dimensional Fe-GIC structure bulit by $\mathrm{C}_{16} \mathrm{Fe}_{4}$ clusters (Fig.3). R. Hoffman and V.Tremela (ref.28) used crystal orbitals method with EHM approximation. Two sets of Fe parameters were used: solid-state set $\left(P_{1}\right)$ and molecular set $\left(P_{2}\right)$. From the obtained data one can conclude that $\pi$-complexes of Fe with carbon layers are formed also in this case. Looking at the atomic orbital populations (Table) you can see that when $P_{1}$. solid-state set was used the charge transfer, occured from Fe atoms to the carbon layers, and when the $P_{2}$ molecular set was used the charge was transferred in the opposite direction. It is rather interesting that the experimental populations obtained from Mossbauer spectroscopy (ref. 9) are in better agreement with calculated values when solid-state set $P_{2}$ was used. So we can summarlze that both approximation support the formation of $\pi$-complexes of $\mathrm{Fe}$ clusters with carbon layers. 


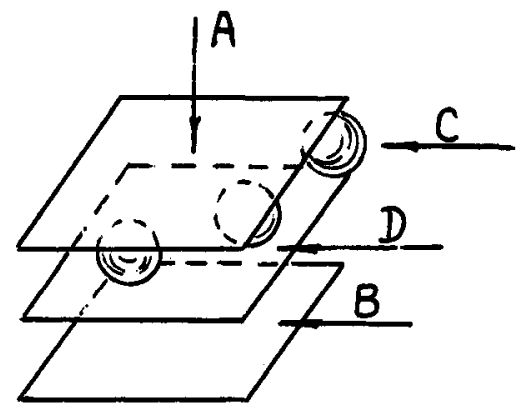

Fig.4. Localisation of possible catalytic centres in GICs

A great variety of bond types in GICs as well as the features of their structure make it possible to expect interesting catalytic properties. For example the interaction between metal atoms and carbon layers could lead to modification and stabilisation of active centers. In addition the conductivity of carbon layers influence the electron transport and catalytic activity. On the other hand the fixed distances between graphite layers might be the reason of catalyst selectivity.

Due to the layred structure of GICs different mechanisms of their catalytic activity can be expected. First, the reactions can proceed at the surfaces of the carbon layers without direct contact between substrate and the intercalated compounds (Figure 4,A). In this case the graphite planes are the catalytic centers of the reaction and the intercalant species are needed to create some charge on them. Second, the catalytic reaction can proceed in the empty interlayer spaces of GICs (B). Now again the catalytic centers are the carbon layers. However, in this case the effect of steric hindrances is crusial since the intercalation of any molecules results with necessity in the significant increase of the interlayer distances. Third, the catalytic reactions can proceed through the direct contact of substrate and intercalated compound either at the edges of the lattice (c), i.e. without penetration of the substrates into interlayer space, or in the filled interlayer spaces of GICs (D). In the last case both the nature of the guest-host interactions and steric factors are of great importance.

The change of intercalant nature provide the possibility to very the GICs catalytic properties. E.g., GICs with metal chlorides are active catalysts of Iiquid-phase oxidation of organic compounds with molecular oxygen (ref.29). According to their activity in the oxidation of ethylbenzene and cumene, the following series can be written for GICs: $\mathrm{CuCl}_{2}(8.3)>\mathrm{MnCl}_{2}(3.8)>\mathrm{CoCl}_{2}(2.8)>$ $>\mathrm{FeCl}_{2}(1.9)>$ graphite (1) $>\mathrm{NICl}_{2}(0.3)>\mathrm{FeCl}_{3}={ }^{2} \mathrm{MoCl}_{5}(0)$. The composition of the products depends on the species of GIC, and if the main product in the case of GIC with $\mathrm{CuCl}_{2}$ is acetophenone, GIC with $\mathrm{MnCl}_{2}$ yields ethylbenzene hydroperoxide.

Depending on their activity in the oxidation of cyclohexene, GICs with metal chlorides form the following series: $\left.\mathrm{CoCl}_{2} \sim \mathrm{CuCl}_{2}(30)>\mathrm{MnCl}_{2}(24)\right\rangle \mathrm{NiCl}_{2}>$ $>\mathrm{FeCl}_{3}(10)>\mathrm{MOCl}_{5}(8)>\mathrm{FeCl}_{2}(6)>\mathrm{ZnCl}_{2}(3)^{2}>\operatorname{graphite}(1)$. It should be noted, however, that GICS are always much more active that the metal chlorides themselves. The kinetic parameters of this reaction indicate that we are dealing with a heterogeneous-homogeneous process, that is the chain arises on the surface of the GIC while its growth occurs in the reaction mixture.

GIC with metal chlorides catalyze also such reactions of electrophilic substitution as alkylation and acylation (refs 32,33 ). In this case, the formation of monoalkylation products take place.

A different type of reactions is associated with GICs with alkaly metals. The negative charge at the graphite plane of these GICs inditates that they must catalyze primarily those reactions which call for electron transfer from the catalyst to the substrate or proceed through the anionic mechanism. This was well demonstrated by Tamaru (refs 32-34).

GICs with potassium are active in the polymerization of some unsaturated compounds (ref.35). Of particular interest is the use of GICs with alkali metals in the polymerization of organocyclosiloxanes. It is known, that the poly- 
merization of organocyclosiloxanes in the presence of alkali yields linear polymers containing an alkali metal in the terminal groups. The presence of such groups in a polymer results in its catalytic depolymerisation and decreases its thermostability. If the polymerization occurs on graphite plane the alkali metal is not transferred to the polymer and the thermal stability of polymers is not decrease. Thus we have found that octamethylcyclotetrasiloxane, hexamethylcyclotrisiloxane, trimethyltriphenylcyclotrisiloxane, and trimethyl-tris-(trifluoropropyl)-cyclotrisiloxane are readily polymerizable in the presence of GICs with alkali metals (refs 36,37 ). According to thermogravimetric and $X$-ray fluorescence analysis data, the polymer does not contain potassium, and its decomposition temperature corresponds to that of a potassium-free polymer. The absence of an alkali metal in the polymer suggests that the latter has a cyclic rather than linear structure. It has been found that in the case of $C_{8} K$ the polymerization proceeds on the surface of $\mathrm{C}_{8} \mathrm{~K}$. But in the case of $\mathrm{C}_{24} \mathrm{k}$ the polymerization proceeds in the interplane space of the GIC.

From the polnt of view of catalysis, GICs with transition metals should be of great interest. The catalytic activity of GICs with transition metals has been studied in reactions of decomposition of alcohols and HCOOH, hydrogenation and isomerization of olefins and acetylenes, reduction of oxygen and oxidation of hydrogen, synthesis of ammonia from nitrogen and hydrogen, and hydrogenation of $\mathrm{CO}$.

The conductivity of GICs make them promising catalysts in electrochemical processes. E.,., it was found that GIC-Co display high catalytic activity in electrochemical reduction of oxygen being more active than well known spinels and silver (ref. 38).

GICs with transition metals (Pt, Pd, Ru, Fe, Co, Ni) display pronounced catalytic activity in the oxidation of hydrogen (ref. 39). In the case of a great excess of oxygen, the reaction is of the first order with respect to hydrogen and zero order with respect to oxygen; the reaction rate is determined by the rate of interaction of $\mathrm{H}_{2}$ with the adsorbed oxygen. The catalytic activity of GICs with transition metals in this case decreases in the series: GIC-Pt $>$ Pd $>\mathrm{Ru}>\mathrm{Ni}>\mathrm{Co}>\mathrm{Fe}$, and varles antibatically with the metal-oxygen bond energy. When catalysis is conducted with excess hydrogen on GIC with platinum metals, characterized by the least energy of the bond with oxygen, the reaction rate is determined by the rate of chemisorption of $\mathrm{O}_{2}$.

GICs catalyse also the dehydrogenation of alcohols and formic acid. The reaction preceeds with high selectivity without any dehydratation and yields aldehydes and ketones and $\mathrm{CO}_{2}$, respectively, maximum activity being achieved when GICs with $\mathrm{Ni}$ and Co have been used (refs 30,40). The high selectivity of Co-GIC was confirmed by the investigation of p-tert. butylcyclohexanol decomposition. Among all possible reaction routes, only one was observed, namely, the dehydrogenation of hydroxyl group which led to exclusively 4-tert. butylcyclohexanone.

A study of the hydrogenation of various unsaturated compounds has not revealed any fundamental difference between GIC-Pd and palladium supported on graphite or carbon. But the introduction of alkali metals into Pd-GIC results in complete deactivation of these catalysts in the reactions of hydrogenation of olefin, thus permits selective hyarogenation of acetylenes to olefins (refs 13, 2.4.).

These results open up new possibilities of regulating the catalytic activity of GICs with transition metals. They include changing the effective charge at the transition metal atoms in the GIC by introducing various additives e.g. the insertion of potassium into GICs with transition metals. We observed this effect of potassium in studying Mossbauer spectra of GIC with Fe and $\mathrm{K}$ (refs $16,41)$.

A study of the synthesis of ammonia from nitrogen and hydrogen catalysing by Fe-GIC has shown that the Fe-GIC alone is not active. The introduction of potassium into the system gives rise to activity which increases with the $K$ : $F e$ ratio and is maximum at $K$ : $F e=6$ to 7 . The same extreme relationship was observed in studying the isotopic exchange of nitrogen (ref. 42.). The experimental data prove a direct relationship between the electronic state of the Fe atom in the GIC and its catalytic activity. These findings agree well with the work of Tamaru (ref. 43) who showed that the system obtained by heating the graphite with metal chloride, followed by treatment with potassium, are 
active in the hydrogenation of $\mathrm{N}_{2}$ to $\mathrm{NH}_{2}$, as well the data reported by Ozaki et al (refs 4.4,4.6) who studied the promoting effect of potassium on the catalytic activity of supported ruthenium. Recently, similar catalysts based on iron carbonyls on potassium-treated carbon supports were found to be active in ammgnia synthesis, the system based on $\mathrm{K}_{2} \mathrm{Fe}_{2}(\mathrm{CO})_{8}$ being perceptibly active at $150^{\circ} \mathrm{C}$ (ref. 47 ).

In the same way GICs with transition metals and potassium catalyze the formation of hydrocarbons from $\mathrm{CO}$ and $\mathrm{H}_{2}$ (ref. 48). In the presence of GICs with Co, Fe, Mo, Os and Ru, methane is primarily formed, while ethane is formed in the presence of GIC with $\mathrm{Cu}$, Pt and Pd. The activity of these systems also increases with potassium to metal ratio. It should be noted that the composition of products of hydrogenation in the presence of cobalt supported on graphite and containing potassium differs considerably from that of the products obtained on GIC with $C o$ and $K$.

The other interesting example is the catalytic activity of GIC with Co and $\mathrm{K}$ in dienes polymerization. This GIC unlike GIC with $\mathrm{K}$ polimerize isoprene selectivity to polyisoprene containing up to $95 \%$ of 3,4-links (ref.49).

The interaction of the inserted substances with the graphite may lead to activation of not only these substances but also the carbon networks of graphite. This may promote transformation of the graphite itself to diamond since this process can be considered as cross-linking of the graphite networks. We have found that GICs with various transition metals are active catalysts of graphite-to-diamond transformation (ref. 50). For example, a mixture of graphite with $10 \%$ Fe-GIC produces diamond at 70 to $80 \mathrm{kbar}$ and 1200 to $1600^{\circ} \mathrm{C}$. This reaction proceeds much faster than in the case of conventional metal catalysts. A study of this process using Mossbauer spectra has revealed that under the reaction condition the GIC with iron remains basically intact and no metalic iron is formed. Thus the formation of GICs lads to mutual modification of both inserted substance and the graphite itself.

In this paper we try to shaw the fruitfulness of the coordination chemistry approach to graphite chemistry. Such approach reveal new possibilities in creations of new materials, new reactions and new catalysts.

\section{REFERENCES}

1. I.P.Vogel, Synth. Metals, 1, 279-86 (1980).

2. Yu.S.Karimov, Yu.N.Novikov and A.V.zvarikina, Phisika Tverdogo Tela, $13,2836-41$ (1971).

3. Yu.S.Karinov, Yu.N.Novikov and M.E.Vol'pin, Pis'ma Zh. Experimental. Teoretich. Phis. 217-20 (1971).

4, M.Elahy, M.Shayegan, K.Y.Szeto and G.Dresselhaus, Synth. Metals, 8, 35-42 (1983).

5. M.E.Vol'pin, Yu.T.Struchkov, Yu.N.Novikov and V.A.Semion, Izv. Acad.Nauk SSSR, Ser.Khim., 2608-9 (1970).

6. H.Klotz and Schneider, Naturwiss., 49, 448 (1962).

7. A.Knappwost and W.Metz, Naturwiss, $53,152-6$ (1966).

8. W.Metz, Dissertation, 19 $\overline{68, \text { Hamburg. }}$

9. M.E.Vol'pin, Yu.N.Novikov, N.D.Lapkina, B.I.Kasatochkin, Yu.T.Struchkov, M.E.Kazakov, R.A.Stukan, V.A.Pavitskil, Yu.S.Karimov and A.V.Zvarikina, J.Amer.Chem., 97, 3366-73 (1975).

10.Yu.N.Novikov, M.E.Kazakov, A.V.Zvarikina, I.S.Astachova, M.E.Vol'pin, Zh. Strukt. Khim., 12, 486-95 (1971).

11. R.A.Stukan, V.E.Prusakov, Yu.N.Novikov, M.E.Vol'pin and V.I.Gol'danskii, Zh.Strukt.Khim., 12, 622-8 (1971).

12. V.E.Prusakov, R.A.Stukan, Yu.N.Novikov, M.E.Vol'pin and V.I.Gol'danskii, Dokl. Akad. Nauk SSSR, 207, 1394-7 (1972).

13.V.A.Prusakov, Ya.V.SaIin, V.I.Nefedov, Yu.N.Novikov and M.E.Vol'pin, Izv.Akad.Nauk SSSR, Ser. Khim., 1689 (1973).

14.A.V.Nefed'ev, R.A.Stukan, V.A.Makarov, V.A.Kondakov, A.T.Schuvaev.N.D. Lapkina, Yu.N.Novikov and M.E.Vol'pin, Zh.Strukt. Khim.,21, 68 (1980).

15.V.G.Jadhao, R.M.Singura, G.M.Joshi, K.P.R.Risharody and C.N.R.Rao, Z.Phys. Chem., $92,139(1974)$. 
16. V.A.Postnikov, A.V.Nefed'ev, Yu.N.Novikov and M.E.Vol'pin, I.zV.Acad.Nauk SSSR, Ser. Khim., $238 \uparrow$ (1975).

17. Ph.Touzain, A.Chamberod and A.Briggs, Mater.Sei..Eng.., 31, 77-81 (1977).

18. C.Ungurenasu and M.Palie, Synth. and React. Inorg. and Metall-organic Chem., 7, 581-9 (1977).

19. D.Braga, A.Ripamonti, D.Savoia, C.Trombini and A.Umani-Ronchi, J.Chem. Soc. Chem. Commun., 927-8 (1978).

20. D.Braga, A.Ripamonti, D.Savoia, C.Trombin and A.Umani-Ronchi, J. Chem. Soc. Dalt. Trans.., 12, 2026-8 (1979).

21. V.L.Solozhenko, A.M.Gus'kov and Yu.A.Kalaschnikov, Dokl. Akad. Nauk SSSR, $273,1143-5(1983)$.

22. P.Scharff and E.Stumpp, Proc. 4-th Intern. Carbon Conf., Baden-Baden, 465 (1986).

23. M.El.Markini, D.Guerard, Ph.Lagrange and A.Herold, Carbon, 18, 203-9 (1980).

24. V.A.Postnikov, Dissertation, Institute of organo-Element Compounds of Academie of Sciences USSR, Moscow, 1977.

25. A.T.Schuvaev, V.A.Kondakov, V.N.Uvarov,. K.Halmoier, N.D.Lapkina, V.A. Postnikov, Yu.N.Novikov, and M.E. Vol'pin, Zh. Strukt. Khim., 20, 736-8 (1979)

26. A.T.Schuvaev, A.P.Kosinov, N.D.Lapkina, Yu.N.Novikov and M.E.Vol'pin, Inorg. Chim. Acta Lett., 54, L35-7 (1981).

27. B.Yu.Hel'mer, T.A.Lyubeznova and V.L.Kraizman, A.C.Mirmil'schtein, L.D. Kvacheva, Yu.N.Novikov and M.E.Vol'pin, IzV.Akad.Nauk SSSR, Ser.Phys., 49, $1471-5$ (1985)

28. R.Hoffman, W.Tremel, M.E.Vol'pin and Yu.N.Novikov, Metalloorgan. Khim., in press.

29. V.M.Belousov, N.I.Kovtyuhova, S.V.Mihailovskii, Yu.N.Novikov and M.E. Vol'pin, Dokl. Akad. Nauk SSSR, 2.53, 346-9 (1980).

30. A.A.Slinkin, Yu.N.Novikov, N.A.Pribytkova, I.I.Lendover, A.M.Rubinshtein and M.E.Vol'pin, Kinetika i Kataliz, 14, 633-6 (1973).

31. J.H.Lalancette, N.J.Fournier-Breault and R.Thiffault, Can.J.Chem., 52, 589-94 (1974).

32. K.Tamaru, Cat. Rev., 4, 161-78 (1979).

33. M.Ichikawa, K. Kawase and K.Tamaru, J.Ehem.Soc., Chem. Commun., 177 (1972).

34. K.Watanade, T.Kondow and K.Tamaru, J.Chem.Soc., Chem.Commun., 39-40 (1972).

35. J.M.Panayaotov and I.B.Rashkov, J.Polym.Sci., 10A, 12.67-70 (1972).

36. Yu.N.Novikov, Ts.V.Kakuliva, I.M.Khananashvili, L.S.Dizeprovskaya, V.M. Kopylov and M.E.Vol'pin, Dokl. Akad. Nauk SSSR, 245, 848-51 (1979).

37. Ts.V.Kakulia, I.M.Khananashvili, L.S.Dneprovskaya, Yu.N.Novikov and M.E. Vol'pin, Vysocomolecular. Soedinenia, 22A, 1690-6 (1980).

38. A.V.Dribinskii, M.P.Tarasevich, N.A.Urison, G.A.Schteinberg, Yu.N.Novikov and M.E.Vol'pin, Electokhim., 13, 1888-9 (1977).

39. N.I.II'chenko, N.P.Maksimovich, Yu.N.Novikov, G.I.Golodets, M.E.Vol'pin, Kinetika i Kataliz, 21, 1463-8 (1980).

40. N.D.Ĺapkina, Yu.N.Novikov and M.E.Vol'pin, Kinetika i Kataliz, 17, 1537-4.1 $(1975)$.

41. V.A.Nefed'ev, R.A.Stukan, V.A.Postnikov, V.B.Shur, Yu.Novikov and M.E. Vol'pin, Izv. Acad. Nauk SSSR, Ser.Khim., 2376-8 (†976).

42. M.E.Vol'pin, Yu.N.Novikov, V.A.Postnikov, V.Shur, B.Bayerl, I.Kaden, M.Waren, I.M.Dmitrienko, R.A.Stukan and A.V.Nefed'ev, z.anorg. allg. Chem.. 9, 231-6 (1977).

43. M.Ichikawa, T.Kondow, K.Kawase, M.Sado, T.Onishi and K.Tamaru, J. Chem. Soc., Chem. Commun., 176-71 (1972).

44. M.I.Ishizuka and A.Ozaki, J.Catal., 35, 320-4 (1972).

45. K.I.Aika, H.Hori and A.Ozaki, J.CataI, 2.7, 424-31. (1972)

46. K.Urube, K.I.Aika and A.Ozaki, J. Cata., 32, 108-13 (1974).

47. V.B.Shur, S.M.Yunusov, V.A.Postnikov, Yu.N.Novikov and M.E.Vol'pin, 3rd Int. Symposium on Relationship Homogeneous and Heteroheneous Catal... Groningen, May 1981, Holland.

48. V.I.Mashinsky, V.A.Postnikov, Yu.N.Novikov, A.I.Lapidus, M.E.Vol 'pin and Ya.T.Eidus, Izv. Akad. Nauk SSSR, Ser.Khim., 9, 2018-23 (1976).

49. G.M.Chernenko, E.I.Tinyakova, Ts.V.Kakulia, I.M.Khananashvili, Yu.N. Novikov and M.E.Vol pin, Vysokomolekular. Soedinenia, 23B, 208-11(1981).

50. Ya.A.Kalashnikov, M.V.Timofeeva, V.I.Faraphontov, V.I.Gol'danskii, R.A. Stukan, A.V.Nefed'ev, M.E.Vol'pin, Yu.N.Novikov and N.D.Lapkina, Synth. Metals, 4, 363-70 (1980). 